\title{
Synthesis and Diels-Alder Reactions of Furo[2,3-c]pyrroles and Benzofuro[2,3-c]pyrroles
}

\author{
Chin-Kang Sha* and Ren-Sheng Lee \\ Department of Chemistry, National Tsing Hua University \\ Hsinchu, Taiwan 300, R. O. C. \\ Yu Wang \\ Department of Chemistry, National Taiwan University \\ Taipei, Taiwan 100, R. O. C.
}

\begin{abstract}
Furo[2,3-c]pyrroles 1a-d and benzofuro[2,3-c]pyrroles 6a-e were synthesized. Diels-Alder reactions of $1 \mathbf{b}$ and $6 \mathbf{b}$ gave $1: 2$ cycloadduct 13 and $1: 1$ cycloadduct 20, respectively. Parent compound 17 of benzofuro[2,3-c]pyrrole ring system was trapped as $N$-tert-butoxycarbonyl derivative 18. Oxidative extrusion of the $N$-bridge in Diels-Alder adduct 20 gave dibenzofuran 22.
\end{abstract}

Furo[2,3-c]pyrroles 1 and benzofuro[2,3-c]pyrroles 6 are highly labile heterocyclic ring systems according to theoretical calculation. ${ }^{1}$ Their synthesis and reaction are unknown. Among the related ring systems 2-5, 7, and 8, only $3^{2}$ and $7^{3}$ were synthesized. However, the method used in these syntheses are unsuitable for the preparation of furo[2,3-c]pyrrole 1 and benzofuro[2,3-c]pyrrole 6 ring systems. We developed three methods for the synthesis of iso-condensed heteroaromatic pyrroles. ${ }^{4}$ In our preliminary communication, we have reported the synthesis of benzo[2,3-c]pyrrole 6.4b Herein we report the preparation of furo[2,3-c]pyrrole 1 and benzofuro[2,3-c]pyrrole 6 in detail, as well as Diels-Alder reactions of these heterocycles.<smiles>[R]n1cc2ccoc2c1</smiles>

1<smiles>[R]n1ccc2ccoc21</smiles>

$\mathbf{2}$<smiles>[R]n1ccc2occc21</smiles>

3<smiles>[R]n1ccc2cocc21</smiles>

4<smiles>[R][N+]1=Cc2cocc2C1</smiles>

5<smiles>[R]n1cc2oc3ccccc3c2c1</smiles>

6<smiles>[R]n1ccc2oc3ccccc3c21</smiles>

7<smiles>[R]n1ccc2c3ccccc3oc21</smiles>

8 
Knoevenagel condensation of 3-methyl-2-furocarboxaldehyde (9) with diethyl malonate gave alkylidenemalonate 10.4 Bromination of 10 with $N$-bromosuccinimide in the presence of dibenzoyl peroxide afforded bromide 11. Treatment of 11 with benzylamine, isopropylamine, tert-butylamine and 3-hydroxypropylamine in ethanol yielded furo[2,3-c]pyrroles 1a, 1b, 1c and 1d, respectively. The yields are only moderate (1646\%) because these compounds are highly sensitive to acid, and partially polymerized upon silica gel chromatography. Subsequently, Diels-Alder reaction of $\mathbf{1 b}$ with two equiv. dimethyl acetylenedicarboxylate (DMAD) at room temperature in benzene gave a 1:2 cycloadduct 13. The expected 1:1 cycloadduct 12 was not detected. Diels-Alder reaction of $1 \mathrm{~b}$ with 0.9 equiv. DMAD also gave only $1: 2$ cycloadduct 13 and recovered 1 b. Apparently, the reactive furan moiety of $1: 1$ cycloadduct 12 underwent a second Diels-Alder reaction with DMAD very rapidly, Scheme 1 . A structure of 13 from $X$-ray crystallographic analysis is shown in Figure $1 .^{5}$

We applied the same method for synthesis of benzofuro[2,3-c]pyrroles 6 . Knoevenagel condensation of 2-methyl-3-benzofurocarboxaldehyde (14) with diethyl malonate gave 15. Bromination of 15 with $N$-bromosuccinimide afforded bromide 16 . Treatment of bromide 16 with methylamine, isopropylamine, benzylamine, phenylamine and p-toluidine afforded benzofuro[2,3-c]pyrroles 6a-e respectively. In addition, treatment of 16 with ammonia in ethanol gave parent compound 17, which was not isolable, but reacted immediately with di-tert-butyl dicarboxylate and 4-dimethylaminopyridine to give stable derivative 18.6 Diels-Alder reactions of compounds 6 and 18 with DMAD afforded cycloadducts 20 and 19 smoothly. $m$-Chloroperbenzoic acid oxidation of 20 with spontaneous extrusion of the $\mathrm{R}-\mathrm{N}=\mathrm{O}$ group gave 3,4-dimethoxycarbonyldibenzofuran (22) via intermediate $\mathbf{2 1 . 7}$

In summary, we have succeeded in synthesis of two new heterocyclic ring systems, furo[2,3-c]pyrrole 1 and benzofuro[2,3-c]pyrrole 6. Diels-Alder reactions of $1 \mathbf{b}$ and $6 \mathbf{b}$ with DMAD gave 1:2 cycloadduct 13 and 1:1 cycloadduct 20 respectively. Oxidative extrusion of the nitrogen bridge in Diels-Alder adduct 20 afforded dibenzofuran 22. Parent system 6 was also prepared and trapped with di-tert-butyl dicarbonate to give 18 , which also underwent Diels-Alder reaction with DMAD.

\section{Experimental Section}

General. ${ }^{1} \mathrm{H}$ NMR spectra were recorded on a Varian EM-390, a JEOL HX-100 or a Bruker AM-400 spectrometer. ${ }^{13} \mathrm{C}$ NMR spectra were recorded on a Bruker AM-400 spectrometer. Mass spectra refer to the electron impact mass spectra and were recorded on a JEOL TMS-D-100 mass spectrometer. High-resolution mass spectra were recorded on 


\section{Scheme 1}

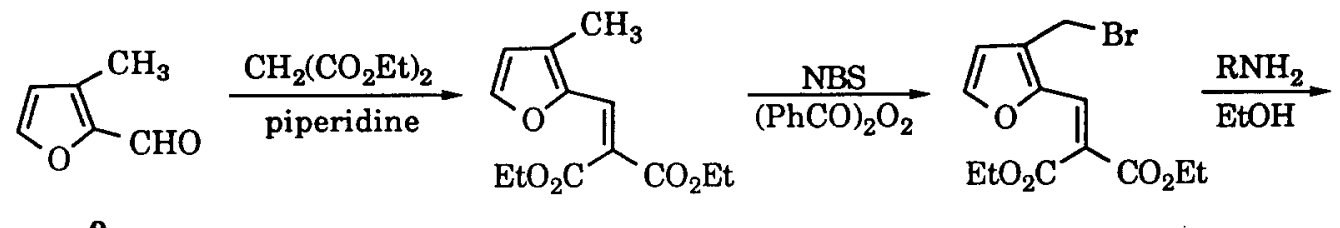

10

11

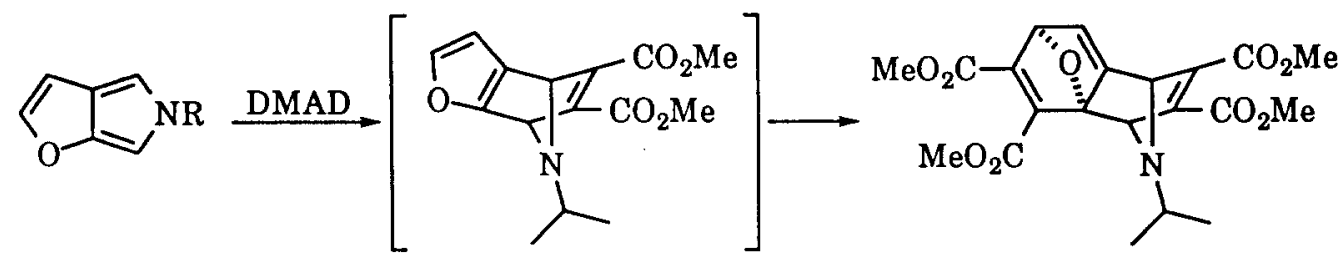

12

13

1a $\mathrm{R}=\mathrm{CH}_{2} \mathrm{Ph}$

1b $\mathrm{R}=\mathrm{CH}\left(\mathrm{CH}_{3}\right)_{2}$

1c $\mathrm{R}=\mathrm{C}\left(\mathrm{CH}_{3}\right)_{3}$

1d $\mathrm{R}=\left(\mathrm{CH}_{2}\right)_{3} \mathrm{OH}$

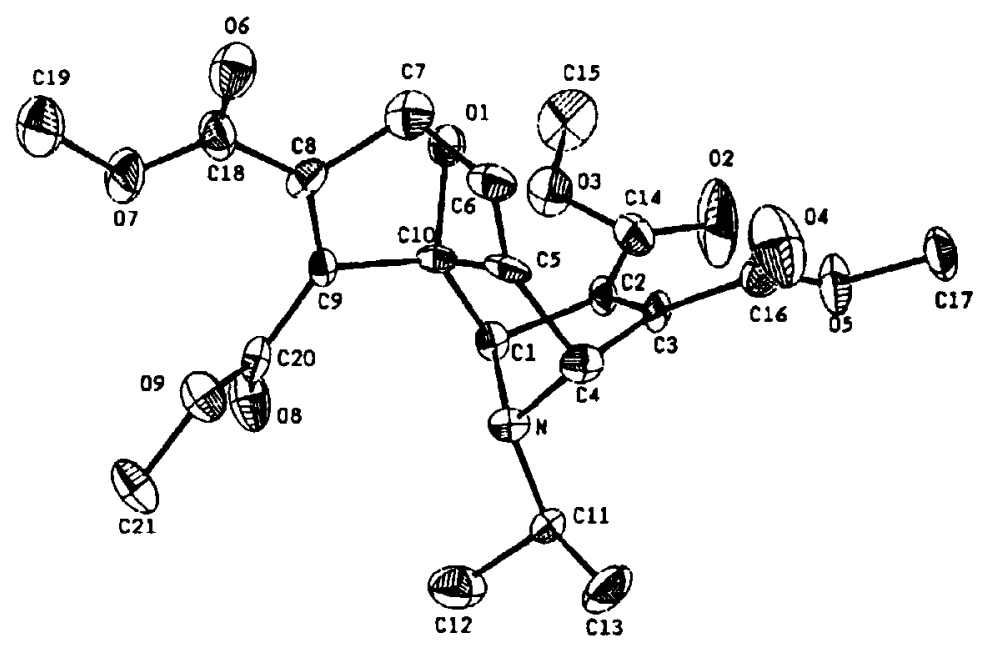

Figure 1 Crystal Structure of 13 


\section{Scheme 2}

14
$\overbrace{\mathrm{OHO}}^{\mathrm{CH}_{3}}$

$$
\underset{\text { piperidine }}{\stackrel{\mathrm{CH}_{2}\left(\mathrm{CO}_{2} \mathrm{Et}\right)_{2}}{\longrightarrow}}
$$

$\mathrm{RNH}_{2}$<smiles>CC(C)(C)[R]1cc2oc3ccccc3c2c1</smiles>

6a $\mathrm{R}=\mathrm{CH}_{3}$

6b R $=\mathrm{CH}\left(\mathrm{CH}_{3}\right)_{2}$

6c $\mathrm{R}=\mathrm{CH}_{2} \mathrm{Ph}$

6d $\mathrm{R}=\mathrm{Ph}$

6e $\mathrm{R}=p-\mathrm{CH}_{3} \mathrm{Ph}$

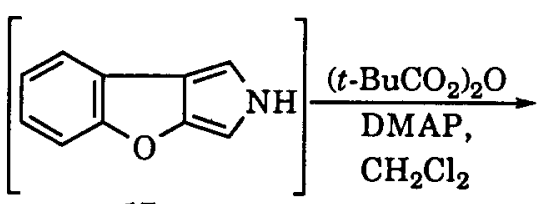

17

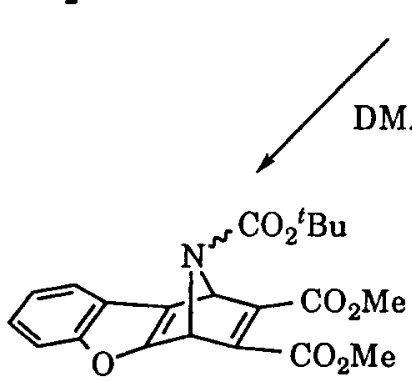

19

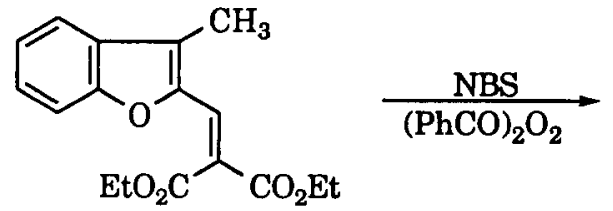

15
$\mathrm{NH}_{3} / \mathrm{EtOH}$<smiles>CCOC(=O)C(=Cc1oc2ccccc2c1CBr)C(=O)OCc1ccccc1</smiles>

16<smiles>CC(C)(N)O</smiles><smiles></smiles>

20 MCPBA

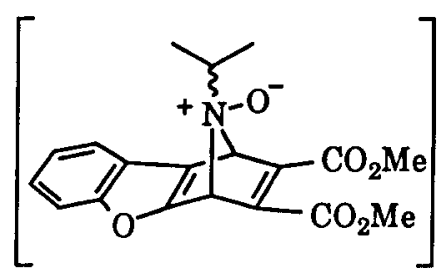

21
$-\mathrm{R}-\mathrm{N}=\mathrm{O}$ 18<smiles>CC(=O)c1cc2oc3ccccc3c2cc1C(C)=O</smiles>

22 
a JEOL HX-110 mass spectrometer. IR spectra were recorded on a Perkin-Elmer 781 spectrometer, and UV spectra on a Perkin-Elmer Lambda 5 UV-VIS spectrometer. Single crystal $X$-ray analysis was performed on a Enraf-Nonius CAD-4 diffractometer. Melting points determined with a Büchi 530 melting-point apparatus and are uncorrected. Flashcolumn chromatography was performed as follows: silica gel, Merck No. 7736 Kieselgel $60 \mathrm{H}$, was placed in a sintered-glass column packed dry. Solvent was flushed through the silica gel under a water-aspirator vacuum. The compound was then deposited with a minimal amount of solvent and eluted with solvent under a water aspirator vacuum. Diethyl ether and tetrahydrofuran (THF) were distilled from potassium/sodium metal under a nitrogen atmosphere with benzophenone ketyl as the indicator. All reactions were conducted under a nitrogen atmosphere.

Diethyl [(3-Bromomethyl-2-furyl)methylene]propanedioate (11). To a solution of 10 (290 $\mathrm{mg}, 1.2 \mathrm{mmol}$ ) in carbon tetrachloride $(25 \mathrm{ml}$ ) was added $N$-bromosuccinimide (204 $\mathrm{mg}, 1.2 \mathrm{mmol})$ and dibenzoyl peroxide $(10 \mathrm{mg})$. The reaction mixture was stirred and heated at reflux for $1 \mathrm{~h}$. After the mixture was cooled in an ice bath, the solid was removed by filtration and washed with carbon tetrachloride. Concentration and silica gel flash column chromatography (hexane-ethyl acetate, 10:1) gave $11(209 \mathrm{mg}, 55 \%)$ as a yellow oil: IR (neat) $\left.2980,1730,1635 \mathrm{~cm}^{-1} ;{ }^{1} \mathrm{H} \mathrm{NMR} \mathrm{(400} \mathrm{MHz} \mathrm{CDCl}_{3}\right) \delta 7.44(\mathrm{~d}, 1 \mathrm{H}, J=1.7 \mathrm{~Hz}), 7.38$ $(\mathrm{s}, 1 \mathrm{H}), 6.49(\mathrm{~d}, 1 \mathrm{H}, J=1.7 \mathrm{~Hz}), 4.56(\mathrm{~s}, 2 \mathrm{H}), 4.34(\mathrm{q}, 2 \mathrm{H}, J=7.2 \mathrm{~Hz}), 4.23(\mathrm{q}, 2 \mathrm{H}, J=7.2$ $\mathrm{Hz}$ ), $1.30(\mathrm{t}, 3 \mathrm{H}, J=7.2 \mathrm{~Hz}), 1.26\left(\mathrm{t}, 3 \mathrm{H}, J=7.2 \mathrm{~Hz}\right.$ ); MS $m / z$ (relative intensity) $322\left(\mathrm{M}^{+}+2\right.$, $44 \%), 330(\mathrm{M}+, 48 \%), 251(100 \%)$.

$N$-Benzylfuro[2,3-c]pyrrole (1a). To a solution of benzylamine (114 mg, $1.1 \mathrm{mmol})$ in $95 \%$ ethanol ( $2 \mathrm{ml})$ was added dropwise a solution of $11(160 \mathrm{mg}, 0.48 \mathrm{mmol})$ in $95 \%$ ethanol $(7 \mathrm{ml})$. The reaction mixture was stirred at room temperature for $31 \mathrm{~h}$. Concentration and silica gel flash column chromatography (hexane-ethyl acetate, 20:1) gave 1a (19mg, 20\%) as a yellow oil: IR (neat) $3120,3030,2930,1605 \mathrm{~cm}^{-1} ;{ }^{1} \mathrm{H} \mathrm{NMR}\left(400 \mathrm{MHz}, \mathrm{CDCl}_{3}\right) \delta 7.34-7.24$ $(\mathrm{m}, 4 \mathrm{H}), 7.12(\mathrm{~s}, 1 \mathrm{H}), 7.11(\mathrm{~d}, 1 \mathrm{H}, J=4.1 \mathrm{~Hz}), 6.50(\mathrm{~d}, 1 \mathrm{H}, J=4.1 \mathrm{~Hz}), 6.49(\mathrm{~s}, 1 \mathrm{H}), 6.40(\mathrm{~s}$, $1 \mathrm{H}), 5.13(\mathrm{~s}, 2 \mathrm{H}) ; \mathrm{MS} m / z$ (relative intensity) $197\left(\mathrm{M}^{+}, 77\right), 91(100)$; HRMS calcd $\mathrm{C}_{13} \mathrm{H}_{11} \mathrm{NO}$ 197.0834 , found 197.0841 .

$N$-Isopropylfuro[2,3-c]pyrrole (1b). To a solution of isopropylamine (38 $\mathrm{mg}, 0.64$ $\mathrm{mmol})$ in $95 \%$ ethanol $(2 \mathrm{ml})$ was added dropwise a solution of $11(96 \mathrm{mg}, 0.29 \mathrm{mmol})$ in $95 \%$ ethanol $(10 \mathrm{ml})$. The reaction mixture was stirred at room temperature for $32 \mathrm{~h}$. Concentration and silica gel flash column chromatography (hexane-ethyl acetate, 20:1) gave $1 \mathrm{~b}(10.8 \mathrm{mg}, 25 \%)$ as a yellow oil: IR (Neat) $3115,2920,1380 \mathrm{~cm}^{-1} ;{ }^{1} \mathrm{H} \mathrm{NMR}(90 \mathrm{MHz}$, $\left.\mathrm{CDCl}_{3}\right) \delta 7.18(\mathrm{bd}, 1 \mathrm{H}), 6.90(\mathrm{bd}, 1 \mathrm{H}), 6.41(\mathrm{~d}, 1 \mathrm{H}, J=3.0 \mathrm{~Hz}), 6.32(\mathrm{~d}, 1 \mathrm{H}, J=3.0 \mathrm{~Hz}), 4.24$ $(\mathrm{m}, 1 \mathrm{H}), 1.45$ (d, $6 \mathrm{H}, J=6.0 \mathrm{~Hz}$ ); MS $m / z$ (relative intensity) $149(\mathrm{M}+100), 107$ (45).

$N$-tert-Butylfuro[2,3-c]pyrrole (1c). To a solution of tert-butylamine (36 $\mathrm{mg}, 0.49$ $\mathrm{mmol})$ in $95 \%$ ethanol $(2 \mathrm{ml})$ was added dropwise a solution of $11(73 \mathrm{mg}, 0.22 \mathrm{mmol})$ in 
95\% ethanol $(10 \mathrm{ml})$. The reaction mixture was stirred and heated at reflex for $24 \mathrm{~h}$. Concentration and silica gel flash column chromatography (hexane-ethyl acetate, 20:1) gave $1 \mathrm{c}(7.1 \mathrm{mg}, 20 \%)$ as a yellow oil: IR (neat) $3120,2940,1640 \mathrm{~cm}^{-1} ;{ }^{1} \mathrm{H}$ NMR $(400 \mathrm{MHz}$, $\left.\mathrm{CDCl}_{3}\right) \delta 7.25(\mathrm{~d}, 1 \mathrm{H}, J=2.1 \mathrm{~Hz}), 6.66(\mathrm{~d}, 1 \mathrm{H}, J=1.6 \mathrm{~Hz}), 6.62(\mathrm{~d}, 1 \mathrm{H}, J=1.6 \mathrm{~Hz}$ ), 6.39 (d, 1 $\mathrm{H}, J=2.1 \mathrm{~Hz}$ ), $1.65\left(\mathrm{~s}, 9 \mathrm{H}\right.$ ); MS $m / z$ (relative intensity) $163\left(\mathrm{M}^{+}, 77\right), 107$ (100).

$\boldsymbol{N}$-(3-Hydroxypropyl)furo[2,3-c]pyrrole (1d). To a solution of 3-hydroxypropylamine (145 $\mathrm{mg}, 1.49 \mathrm{mmol})$ in $95 \%$ ethanol $(2 \mathrm{ml})$ was added dropwise a solution of 11 (128 $\mathrm{mg}$, $0.39 \mathrm{mmol}$ ) in $95 \%$ ethanol $(10 \mathrm{ml})$. The reaction mixture was stirred at room temperature for $18 \mathrm{~h}$. Concentration and silica gel flash column charomatography (hexane-ethyl acetate, $5: 1)$ gave $1 \mathrm{~d}\left(6 \mathrm{mg}, 10 \%\right.$ ) as a yellow oil: IR (neat) $3415,3010,1376 \mathrm{~cm}^{-1} ; 1 \mathrm{H} \mathrm{NMR}$ $\left(90 \mathrm{MHz}^{\mathrm{CDCl}} 3\right) \delta 7.26(\mathrm{~d}, 1 \mathrm{H}, J=1.5 \mathrm{~Hz}), 6.48(\mathrm{~d}, 1 \mathrm{H}, J=1.5 \mathrm{~Hz}), 6.42(\mathrm{~d}, 1 \mathrm{H}, J=2.4 \mathrm{~Hz}$ ), $6.36(\mathrm{~d}, 1 \mathrm{H}, J=2.4 \mathrm{~Hz}), 4.11(\mathrm{t}, 2 \mathrm{H}, J=6.0 \mathrm{~Hz}), 4.61(\mathrm{t}, 2 \mathrm{H}, J=6.0 \mathrm{~Hz}), 2.09$ (quintet, $2 \mathrm{H}, J$ $=6.0 \mathrm{~Hz}$ ), $1.59(\mathrm{bs}, 1 \mathrm{H})$; MS $m / z$ (relative intensity) $165\left(\mathrm{M}^{+}, 100\right), 121(54)$.

Cycloadduct 13 of $N$-Isopropylfuro[2,3-c]pyrrole (1b) and Dimethyl Acetylenedicarboxylate. A solution of $1 \mathrm{~b}(6 \mathrm{mg}, 0.04 \mathrm{mmol})$ and dimethyl acetylenedicarboxylate $(11.4 \mathrm{mg}, 0.08 \mathrm{mmol})$ in benzene $(3 \mathrm{ml})$ were stirred at room temperature for $5 \mathrm{~h}$. Concentration and silica gel flash column chromatography (hexaneethyl acetate, $5: 1)$ gave $13\left(15.3 \mathrm{mg}, 88 \%\right.$ ) as a white solid: $\mathrm{mp} 107-108^{\circ} \mathrm{C}$; IR (neat) 3130 , $1732,1718,1635 \mathrm{~cm}^{-1}$; ${ }^{1} \mathrm{H} \mathrm{NMR}\left(400 \mathrm{MHz} \mathrm{CDCl}_{3}\right) \delta 6.47(\mathrm{~s}, 1 \mathrm{H}), 5.67$ (d, $\left.1 \mathrm{H}, J=1.4 \mathrm{~Hz}\right)$, $5.01(\mathrm{~s}, 1 \mathrm{H}), 4.98(\mathrm{~s}, 1 \mathrm{H}), 3.81(\mathrm{~s}, 3 \mathrm{H}), 3.80(\mathrm{~s}, 3 \mathrm{H}), 3.79(\mathrm{~s}, 3 \mathrm{H}), 3.77(\mathrm{~s}, 3 \mathrm{H}), 2.76(\mathrm{~m}, 1 \mathrm{H})$, 0.97 (d, $3 \mathrm{H}, J=6.2 \mathrm{~Hz}$ ), 0.94 (d, $3 \mathrm{H}, J=6.2 \mathrm{~Hz}$ ); 13C NMR (100.6 MHz, CDCl3) $\delta 163.70(\mathrm{~s})$, 163.48 (s), 163.29 (s), 162.40 (s), 157.31 (s), 151.09 (s), 149.58 (s), 143.10 (s), 140.52 (s), 126.21 (s), 98.59 (s), 87.20 (d), 66.33 (d), 65.91 (d), 52.50 (q), 52.42 (q), 52.19 (q), 52.11 (q), 45.40 (q), 22.01 (q), 21.90 (q); MS $m / z$ (relative intensity) $433\left(\mathrm{M}^{+}, 27\right), 401$ (100), 358 (27); HRMS calcd $\mathrm{C}_{21} \mathrm{H}_{23} \mathrm{NO}_{9} 433.1376$, found 433.1373 .

Diethyl [(3-Methyl-2-benzofuryl)methylene]propanedioate (15). To a solution of 14 $(1 \mathrm{~g}, 6.25 \mathrm{mmol})$ in dry benzene $(30 \mathrm{ml})$ was added diethyl malonate $(3 \mathrm{~g}, 18.75 \mathrm{mmol})$, piperidine $(0.15 \mathrm{ml})$ and acetic acid $(0.1 \mathrm{ml})$. The reaction mixture was refluxed for $26 \mathrm{~h}$ with a Dean-Stark water separator attached. After the mixture were washed with $\mathrm{H}_{2} \mathrm{O}(20$ $\mathrm{ml} \times 2), 5 \%$ hydrochloride acid $(10 \mathrm{ml} \times 2)$, saturated sodium carbonate $(15 \mathrm{ml})$ and then dried $\left(\mathrm{MgSO}_{4}\right)$. Concentration and silica gel flash column chromatography (hexane-ethyl acetate, 10:1) gave $15(1.76 \mathrm{~g}, 94 \%)$ as a yellow solid: $\mathrm{mp} 89-90^{\circ} \mathrm{C}$; $\mathrm{IR}(\mathrm{KBr}) 2965,1745,1690$, $1620 \mathrm{~cm}^{-1} ;{ }^{1} \mathrm{H} \mathrm{NMR}\left(90 \mathrm{MHz}, \mathrm{CDCl}_{3}\right) \delta 7.61(\mathrm{~s}, 1 \mathrm{H}), 7.40-7.12(\mathrm{~m}, 4 \mathrm{H}), 4.45(\mathrm{q}, 2 \mathrm{H}, J=6.8$ $\mathrm{Hz}), 4.28(\mathrm{q}, 2 \mathrm{H}, J=6.8 \mathrm{~Hz}), 2.41(\mathrm{~s}, 3 \mathrm{H}), 1.41(\mathrm{t}, 3 \mathrm{H}, J=6.8 \mathrm{~Hz}), 1.30(\mathrm{t}, 3 \mathrm{H}, J=6.8 \mathrm{~Hz}$ ); MS $m / z$ (relative intensity) $302(\mathrm{M}+, 93), 256(100), 184$ (33); HRMS calcd $\mathrm{C}_{17} \mathrm{H}_{18} \mathrm{O}_{5} 302.1149$, found 302.1154 .

Diethyl [(3-Bromomethyl-2-benzofuryl)methylene]propanedioate (16). To a solution of $15(1.65 \mathrm{~g}, 5.4 \mathrm{mmol})$ in carbon tetrachloride $(50 \mathrm{ml})$ was added $N$-bromosuccinimide 
$(970 \mathrm{mg}, 5.4 \mathrm{mmol})$ and dibenzoyl peroxide $(80 \mathrm{mg})$. The reaction mixture was stirred and heated at reflux for $1 \mathrm{~h}$. After the mixture was cooled in an ice bath, the solid was removed by filtration and washed with carbon tetrachloride. Concentration and silica gel flash column chromatography (hexane-ethyl acetate, 10:1) gave $16(1.85 \mathrm{~g}, 90 \%)$ as a yellow solid: $\mathrm{mp} 115-116^{\circ} \mathrm{C}$; IR (KBr) $2985,1732,1718,1636 \mathrm{~cm}^{-1} ;{ }^{1} \mathrm{H}$ NMR (400 $\left.\mathrm{MHz} \mathrm{CDCl}_{3}\right) \delta 7.67$ (dd, $1 \mathrm{H}, J=7.2,0.8 \mathrm{~Hz}$ ), 7.41-7.27 (m, $4 \mathrm{H}$ ), $4.69(\mathrm{~s}, 2 \mathrm{H}), 4.46(\mathrm{q}, 2 \mathrm{H}, J=7.3 \mathrm{~Hz}), 4.31(\mathrm{q}, 2$ $\mathrm{H}, J=7.3 \mathrm{~Hz}), 1.41(\mathrm{t}, 3 \mathrm{H}, J=7.3 \mathrm{~Hz}), 1.33(\mathrm{t}, 3 \mathrm{H}, J=7.3 \mathrm{~Hz}) ;{ }^{13} \mathrm{C} \mathrm{NMR}\left(100.6 \mathrm{MHz}, \mathrm{CDCl}_{3}\right)$ $\delta 165.70$ (s), 163.71 (s), 155.29 (s), 147.21 (s), 127.78 (d), 126.80 (s), 125.91 (s), 123.70 (d), 123.63 (d), 123.00 (s), 120.68 (d), 111.59 (d), 62.01 (t), 61.80 (t), 20.01 (t), 14.09 (q), 14.02 (q); MS m/z (relative intensity) $382\left(\mathrm{M}^{+}+2,26\right), 380\left(\mathrm{M}^{+}, 26\right), 301(100), 255$ (35); HRMS calcd $\mathrm{C}_{17} \mathrm{H}_{17} \mathrm{BrO}_{5}$ 380.0259 , found 380.0224 .

N-Methylbenzofuro[2,3-c]pyrrole (6a). To a solution of $35 \%$ methylamine (29.7 $\mathrm{mg}$, $0.96 \mathrm{mmol}$ ) in $95 \%$ ethanol ( $2 \mathrm{ml}$ ) was added dropwise a solution of 16 (166 mg, $0.44 \mathrm{mmol}$ ) in $95 \%$ and ethanol-tetrahydrofuran $(1: 1,6 \mathrm{ml})$. The reaction mixture was stirred at room emperature for $16 \mathrm{~h}$. Concentration and silica gel flash column chromatography (hexaneethyl acetate, $20: 1)$ gave $6 \mathrm{a}(41 \mathrm{mg}, 55 \%)$ as a white solid: $\mathrm{mp} 102-103^{\circ} \mathrm{C}$; IR (KBr) 3118 , 2935, 1635, 1580, $1385 \mathrm{~cm}^{-1} ;{ }^{1} \mathrm{H}$ NMR (400 MHz, $\left.\mathrm{CDCl}_{3}\right) \delta 7.63$ (dd, $1 \mathrm{H}, J=7.3,1.0 \mathrm{~Hz}$ ), 7.40 $(\mathrm{d}, 1 \mathrm{H}, J=7.3 \mathrm{~Hz}$ ), $7.22(\mathrm{~m}, 2 \mathrm{H}), 6.72(\mathrm{~d}, 1 \mathrm{H}, J=1.3 \mathrm{~Hz}), 6.49(\mathrm{~d}, 1 \mathrm{H}, J=1.3 \mathrm{~Hz}), 3.77(\mathrm{~s}, 3$ $\mathrm{H}) ;{ }^{13} \mathrm{C} \mathrm{NMR}\left(100.6 \mathrm{MHz} \mathrm{CDCl}_{3}\right.$ ) $\delta 161.10$ (s), 150.29 (s), 121.41 (d), 122.70 (s), 122.01 (d), 120.33 (d), 114.74 (s), 111.39 (d), 108.50 (d), 97.58 (d), 37.40 (q); MS $m / z$ (relative intensity) 171 $\left(\mathrm{M}^{+}, 100\right)$; HRMS calcd $\mathrm{C}_{11} \mathrm{H}_{9} \mathrm{NO} 171.0684$, found 171.0674 .

$N$-Isopropylbenzofuro[2,3-c]pyrrole (6b). To a solution of isopropylamine (38 $\mathrm{mg}$, $0.64 \mathrm{mmol})$ in $95 \%$ ethanol $(2 \mathrm{ml})$ was added dropwise a solution of $16(110 \mathrm{mg}, 0.29 \mathrm{mmol})$ in $95 \%$ ethanol-tetrahydrofuran $(2: 1,15 \mathrm{ml})$. The reaction mixture was stirred at room temperature for $18 \mathrm{~h}$. Concentration and silica gel flash column chromatography (hexane-ethyl acetate, $20: 1)$ gave $6 \mathrm{~b}(34 \mathrm{mg}, 80 \%)$ as a white solid: $\mathrm{mp} 54-55^{\circ} \mathrm{C}$; IR ( $\mathrm{KBr}$ ) $2975,1625,1570,1405,1370 \mathrm{~cm}^{-1} ;{ }^{1} \mathrm{H} \mathrm{NMR}\left(90 \mathrm{MHz} \mathrm{CDCl}_{3}\right) \delta 7.53(\mathrm{~m}, 1 \mathrm{H}), 7.39-7.04(\mathrm{~m}, 3$ $\mathrm{H}), 6.81(\mathrm{~d}, 1 \mathrm{H}, J=2.7 \mathrm{~Hz}), 6.51(\mathrm{~d}, 1 \mathrm{H}, J=2.7 \mathrm{~Hz}), 4.25(\mathrm{~m}, 1 \mathrm{H}), 1.56(\mathrm{~d}, 6 \mathrm{H}, J=6.0 \mathrm{~Hz})$; MS $m / z$ (relative intensity) $199\left(\mathrm{M}^{+}, 100\right)$; HRMS calcd $\mathrm{C}_{13} \mathrm{H}_{13} \mathrm{NO} 199.0998$, found 199.0997.

$N$-Benzylbenzofuro[2,3-c]pyrrole (6c). To a solution of benzylamine (102 $\mathrm{mg}, 0.95$ $\mathrm{mmol}$ ) in 95\% ethanol ( $2 \mathrm{ml})$ was added dropwise a solution of $16(165 \mathrm{mg}, 0.43 \mathrm{mmol}$ ) in $95 \%$ ethanol-tetrahydrofuran $(1: 1,6 \mathrm{ml})$. The reaction mixture was stirred at room temperature for $22 \mathrm{~h}$. Concentration and silica gel flash column chromatography (hexane-ethyl acetate, $20: 1)$ gave $6 \mathrm{c}(84.5 \mathrm{mg}, 79 \%)$ as a white solid: $\mathrm{mp} 88-89^{\circ} \mathrm{C}$; IR (KBr) $3025,1630,1580,1395,1198 \mathrm{~cm}^{-1} ;{ }^{1} \mathrm{H}$ NMR (400 MHz, $\left.\mathrm{CDCl}_{3}\right) \delta 7.64(\mathrm{~d}, 1 \mathrm{H}, J=7.4 \mathrm{~Hz}$ ), 7.41 $(\mathrm{d}, 1 \mathrm{H}, J=7.4 \mathrm{~Hz}), 7.36-7.15(\mathrm{~m}, 7 \mathrm{H}), 6.85(\mathrm{~d}, 1 \mathrm{H}, J=1.4 \mathrm{~Hz}), 6.57(\mathrm{~d}, 1 \mathrm{H}, J=1.4 \mathrm{~Hz}), 5.16$ (s, $2 \mathrm{H}$ ); 13C NMR (100.6 MHz, $\mathrm{CDCl}_{3}$ ) $\delta 161.40$ (s), 150.50 (s), 138.01 (s), 128.90 (d, 2c), 127.91 (d), 127.12 (d, 2c), 124.50 (d), 122.73 (s), 122.20 (d), 120.59 (d), 115.09 (s), 111.61 (d), 108.19 (d), 
97.40 (d), 54.81 (t); MS $m / z$ (relative intensity) $247\left(\mathrm{M}^{+}, 100\right.$ ); HRMS calcd $\mathrm{C}_{17} \mathrm{H}_{13} \mathrm{NO}$ 247.0998, found 247.0982.

$N$-Phenylbenzofuro[2,3-c]pyrrole (6d). To a solution of aniline $(88.7 \mathrm{mg}, 0.95 \mathrm{mmol})$ in $95 \%$ ethanol $(2 \mathrm{ml}$ ) was added dropwise a solution of 16 (165 $\mathrm{mg}, 0.43 \mathrm{mmol}$ ) in $95 \%$ ethanol-tetrahydrofuran $(1: 1,6 \mathrm{ml})$. The reaction mixture was stirred and heated at $65^{\circ} \mathrm{C}$ for $20 \mathrm{~h}$. Concentration and silica gel flash column chromatography (hexane-ethyl acetate, 20:1) gave 6d (39.6 mg, 17\%). IR (KBr) 3031, 1640, 1382, 1220, $745 \mathrm{~cm}^{-1}$; ${ }^{1} \mathrm{H} \mathrm{NMR}$ (400 $\left.\mathrm{MHz} \mathrm{CDCl}_{3}\right) \delta 7.67(\mathrm{~d}, 1 \mathrm{H}, J=7.5 \mathrm{~Hz}), 7.45(\mathrm{~d}, 4 \mathrm{H}, J=4.2 \mathrm{~Hz}), 7,39(\mathrm{~d}, 1 \mathrm{H}, J=7.5 \mathrm{~Hz}), 7.27$ (m, $2 \mathrm{H}$ ), $7.20(\mathrm{~m}, 1 \mathrm{H}), 7.20(\mathrm{~d}, 1 \mathrm{H}, J=1.4 \mathrm{~Hz}), 6.95(\mathrm{~d}, 1 \mathrm{H}, J=1.4 \mathrm{~Hz}$ ); MS $\mathrm{m} / z$ (relative intensity) $233\left(\mathrm{M}^{+}, 100\right)$; HRMS calcd $\mathrm{C}_{16} \mathrm{H}_{11} \mathrm{NO} 233.0851$, found 233.0840 .

$N \cdot p$-Tolylbenzofuro[2,3-c]pyrrole (6e). To a solution of p-toluidine (98 $\mathrm{mg}, 0.91$ $\mathrm{mmol}$ ) in $95 \%$ ethanol ( $2 \mathrm{ml}$ ) was added dropwise a solution of $16(134 \mathrm{mg}, 0.35 \mathrm{mmol})$ in 95\% ethanol $(6 \mathrm{ml})$. The reaction mixture was stirred at room temperature for $34 \mathrm{~h}$. Concentration and silica gel flash column chromatography (hexane-ethyl acetate, 20:1) gave $6 \mathrm{e}(17.2 \mathrm{mg}, 20 \%)$ as a yellow solid: $\mathrm{mp} 137-138^{\circ} \mathrm{C}$; IR $(\mathrm{KBr}) 3008,1645,1380 \mathrm{~cm}^{-1} ;{ }^{1} \mathrm{H}$ $\operatorname{NMR}\left(400 \mathrm{MHz}, \mathrm{CDCl}_{3}\right) \delta 7.67(\mathrm{~d}, 1 \mathrm{H}, J=6.8 \mathrm{~Hz}), 7.39(\mathrm{~d}, 1 \mathrm{H}, J=7.8 \mathrm{~Hz}), 7.34(\mathrm{~d}, 2 \mathrm{H}, J=$ $6.8 \mathrm{~Hz}$ ), $7.25(\mathrm{~m}, 3 \mathrm{H}), 7.20$ (d, $1 \mathrm{H}, J=7.8 \mathrm{~Hz}), 7.16(\mathrm{~d}, 1 \mathrm{H}, J=1.9 \mathrm{~Hz}), 6.91(\mathrm{~d}, 1 \mathrm{H}, J=1.9$ $\mathrm{Hz}$ ), $2.38\left(\mathrm{~s}, 3 \mathrm{H}\right.$ ); MS $m / z$ (relative intensity) $247\left(\mathrm{M}^{+}, 100\right), 232$ (31); HRMS calcd for $\mathrm{C}_{17} \mathrm{H}_{13} \mathrm{NO} 247.1003$, found 247.0997.

$N$-tert-Butoxycarbonylbenzofuro[2,3-c]pyrrole (18). To a solution of 16 (78 $\mathrm{mg}, 0.21$ $\mathrm{mmol})$ in $95 \%$ ethanol $(10 \mathrm{ml})$ was added dropwise $25 \%$ ammonia water $(0.15 \mathrm{ml})$. The reaction mixture was stirred at room temperature for $52 \mathrm{~h}$. After concentration, a solution of 4-dimethylaminopyridine $(50 \mathrm{mg}, 0.41 \mathrm{mmol})$, di-tert-butyl dicarbonate (134 mg, $0.61 \mathrm{mmol})$ in dry dichloromethane $(10 \mathrm{ml})$ was added. The reaction mixture was stirred at room temperature for $2 \mathrm{~h}$. Concentration and silica gel flash column chromatography (hexane-ethyl acetate, $5: 1$ ) gave $18(12.6 \mathrm{mg}, 25 \%$ ) as a yellow oil: IR (neat) $2980,1740,1415$, $1360,1250,1160 \mathrm{~cm}^{-1} ;{ }^{1} \mathrm{H} \mathrm{NMR}\left(90 \mathrm{MHz} \mathrm{CDCl}_{3}\right.$ ) $\delta 7.66$ (dd, $1 \mathrm{H}, J=6.6,1.5 \mathrm{~Hz}$ ), 7.40-7.18 $(\mathrm{m}, 4 \mathrm{H}), 7.04(\mathrm{~d}, 1 \mathrm{H}, J=1.5 \mathrm{~Hz}), 1.65(\mathrm{~s}, 9 \mathrm{H}) ; \mathrm{MS} m / z$ (relative intensity) $257\left(\mathrm{M}^{+}, 56\right), 201$ (100), 157 (63); HRMS calcd for $\mathrm{C}_{15} \mathrm{H}_{15} \mathrm{NO}_{3} 257.1053$, found 257.1051.

Cycloadduct 19 of $N$-tert-butoxycarbonylbenzofuro[2,3-c]pyrrole (18) and Dimethyl Acetylenedicarboxylate. A solution of $18(14 \mathrm{mg}, 0.05 \mathrm{mmol})$ and dimethyl acetylenedicarboxylate $(15.5 \mathrm{mg}, 0.11 \mathrm{mmol})$ in benzene $(5 \mathrm{ml})$ were refluxed for $9 \mathrm{~h}$. Concentration and silica gel flash column chromatography (hexane-ethyl acetate, 7:1) gave 19 (17.8mg, $82 \%$ ) as a yellow oil: IR (neat) $2945,1770-1710,1530,1385,1255 \mathrm{~cm}^{-1} ;{ }^{1} \mathrm{H}$ NMR (90 MHz, $\left.\mathrm{CDCl}_{3}\right) \delta 7.75(\mathrm{~s}, 2 \mathrm{H}), 7.30(\mathrm{~s}, 2 \mathrm{H}), 3.78(\mathrm{~s}, 6 \mathrm{H}), 3.69(\mathrm{~m}, 2 \mathrm{H}), 1.57(\mathrm{~s}, 9 \mathrm{H})$; MS $m / z$ (relative intensity) $399\left(\mathrm{M}^{+}, 24\right), 283(50), 227(32), 183$ (100); HRMS calcd for $\mathrm{C}_{21} \mathrm{H}_{21} \mathrm{NO}_{7} 399.1318$, found 399.1330. 
Cycloadduct 20 of $N$-isopropylbenzofuro[2,3-c]pyrrole (6b) and Dimethyl Acetylenedicarboxylate. A solution of $6 \mathrm{~b}(18 \mathrm{mg}, 0.09 \mathrm{mmol})$ and dimethyl acetylenedicarboxylate $(15.4 \mathrm{mg}, 0.11 \mathrm{mmol})$ in benzene $(5 \mathrm{ml})$ were stirred at room temperature for $60 \mathrm{~h}$. Concentration and silica gel flash column chromatography (hexane-ethyl acetate, 5:1) gave $20(22.9 \mathrm{mg}, 75 \%$ ) as a yellow oil: IR (neat) 1950, 1740, 1715, $1580,1572,1432 \mathrm{~cm}^{-1} ;{ }^{1} \mathrm{H}$ NMR (90 MHz, $\mathrm{CDCl}_{3}$ ) $\delta$ 7.50-7.30 (m, $2 \mathrm{H}$ ), 7.28-7.08 (m, $2 \mathrm{H}$ ), 5.55 (d, $1 \mathrm{H}, J=0.2 \mathrm{~Hz}$ ), 5.09 (d, $1 \mathrm{H}, J=0.2 \mathrm{~Hz}$ ), 3.79 (s, $3 \mathrm{H}$ ), $3.72(\mathrm{~s}, 3 \mathrm{H}), 2.88(\mathrm{~m}, 1 \mathrm{H}), 1.10$ (d, $6 \mathrm{H}, J=6.0 \mathrm{~Hz}$ ); MS $m / z$ (relative intensity) $341\left(\mathrm{M}^{+}, 90\right), 225(100), 199(70)$.

Dimethyl Dibenzofuran-2,3-dicarboxylate (22). To a solution of $20(22 \mathrm{mg}, 0.06 \mathrm{mmol})$ in dichloromethane $(5 \mathrm{ml})$ was added dropwise a solution of $m$-chloroperbenzoic acid (15.3 $\mathrm{mg}, 0.07 \mathrm{mmol}$ ) in dichloromethane $(3 \mathrm{ml}$ ). The reaction mixture was stirred at room temperature for $3 \mathrm{~h}$. The reaction mixture was then washed with saturated sodium thiosulfate solution, water and brine. Concentration and silica gel flash column chromatography (hexane-ethyl acetate, $2: 1)$ gave $22(9.5 \mathrm{mg}, 56 \%)$ as a yellow oil: IR (neat) $3100,2900,1720,1640 \mathrm{~cm}^{-1}$; ${ }^{1} \mathrm{H}$ NMR (400 $\left.\mathrm{MHz} \mathrm{CDCl}_{3}\right) \delta 8.36(\mathrm{~s}, 1 \mathrm{H}), 7.98$ (d, $1 \mathrm{H}, J=7.6$ $\mathrm{Hz}$ ), $7.88(\mathrm{~s}, 1 \mathrm{H}), 7.61(\mathrm{~d}, 1 \mathrm{H}, J=8.3 \mathrm{~Hz}$ ), 7.54 (dd, $1 \mathrm{H}, \mathrm{J}=8.3,7.2 \mathrm{~Hz}$ ), 7.40 (dd, $1 \mathrm{H}, J=7.6$, $7.2 \mathrm{~Hz}$ ), $3.9(\mathrm{~s}, 6 \mathrm{H}) ; \mathrm{MS} m / z$ (relative intensity) $284\left(\mathrm{M}^{+}, 100\right), 253(26), 236(27)$; HRMS calcd for $\mathrm{C}_{16} \mathrm{H}_{12} \mathrm{O}_{5} 284.0685$, found 284.0684 .

Acknowledgement: We thank the National Science Council of the Rupublic of China for the financial support (NSC-83-0208-M007-028).

\section{References and Notes:}

1. Milum, M.; Trinajustic, N. Croa. Chem. Acta. 1977, 107.

2. Soth, S.; Farnier, M.; Paulmier, C. Can. J. Chem. 1978, 56, 1429.

3. Saruwatari, M.; Hatano, S.; Isomura, K.; Taniguchi, H.; Fukusokan Kagaku Toronkai Koen Yoshishu 12th 1979, 211. C. A. 1980, 93, 95115y; Krutosikova, A.; Kovac, J.; Dandarova, M.; Bobalova, M.; Collect. Czech. Chem. Commun. 1982, 47 (12), 3288.

4. (a) Sha, C.-K.; Tsou, C.-P. J. Org. Chem. 1990, 55, 2446. (b) Sha, C.-K.; Tsou, C.-P, Li, Y.-C.; Lee, R.-S.; Tsai, F.-Y.; Yeh, R.-H. J. Chem. Soc,, Chem. Commun. 1988, 1081. (c) Sha, C.-K.; Tsou, C.-P. J. Chem. Soc., Chem. Commun. 1986, 310.

5. Crystal data of 13: $\mathrm{C}_{21} \mathrm{H}_{23} \mathrm{NO}_{9}: \mathrm{M}=433.41$, monoclinic, space group $\mathrm{P} 22_{1 / \mathrm{c}}, \mathrm{a}=9.819(7)$, $b=8.906(4), c=23.503(7) \AA, \alpha=90^{\circ}, \beta=91.72^{\circ}, Z=4.2712$ Unique reflections were measured of which 892 were considered observed [I $>1.5 \sigma(\mathrm{I})]$. The structure was solved by direct 
method to an $R$ value of 0.066 . All calculations were performed with the NRCC-SDP package. The supplementary materials have been deposited at the Cambridge Crystallographic Data Center.

6. Grieco, P. A.; Flynn, D. L.; Zelle, R. E. J. Org. Chem. 1983, 48, 2424.

7. Gribble, G. W.; Allen, R. W. Tetrahedron Lett. 1976, 17, 3673.

(Received in China 1 August 1994; accepted 25 September 1994) 